\title{
Crossover Iterated Local Search for SDCARP
}

\author{
An-Yang Liang • Dan Lin
}

Received: 18 February 2014/Revised: 29 May 2014/Accepted: 13 August 2014/

Published online: 12 September 2014

(C) Operations Research Society of China, Periodicals Agency of Shanghai University, and SpringerVerlag Berlin Heidelberg 2014

\begin{abstract}
This paper introduces a new algorithm based on local search for the capacitated arc routing problem (CARP) and the split-delivery capacitated arc routing problem (SDCARP). We present a intermediate model to transfer CARP to SDCARP and then solve the two problems by an algorithm which combines the iterated local search and the memetic algorithm. We use crossovers to perform fully reproducible initializations in each local search iteration and edge-marking to save computation time. The computational results on 63 instances of standard benchmarks show that the proposed algorithm outperforms most of the existing best-known solutions obtained by other heuristics within a reasonable computing time. Furthermore, compared with the CARP solutions, our algorithm finds three optimums for the SDCARP.
\end{abstract}

Keywords Capacitated arc routing problem - Split-delivery $\cdot$ Memetic algorithm . Iterated local search

\section{Introduction}

The capacitated arc routing problem (CARP) can be stated as follows [13] : Let $G$ be an undirected graph $G=(V, E)$, where $V=\left\{v_{0}, v_{1} \cdots, v_{n}\right\}$ is a node set and $E=\left\{\left[v_{i}, v_{j}\right]: v_{i}, v_{j} \in V, i<j\right\}$ is an edge set, each edge of $E$ has a positive cost or length $C_{e}$ and nonnegative demand or weight $D_{e}$. Node $v_{0}$ represents a depot node at which a fleet of identical vehicles of capacity $W\left(W \geqslant \max \left\{D_{e}, e \in E\right\}\right)$ are based. The number of vehicles is a decision variable. The aim is to find a set of vehicle

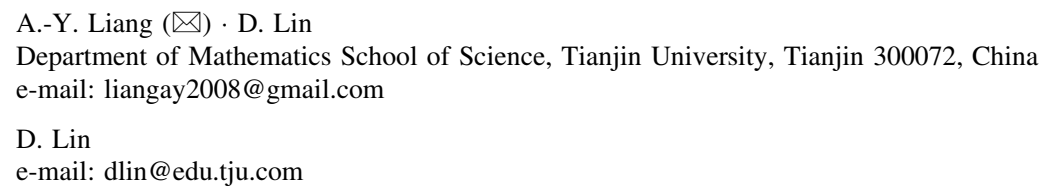


routes of total minimum cost such that: (1) each route starts and ends at the depot; (2) each positive-demand edge is serviced by exactly one vehicle; (3) the total demand of each route does not exceed the vehicle's capacity $W$. Furthermore, each edge $e$ with $D_{e}>0$, called a required edge, must be serviced by a vehicle. On the other hand, each edge $e$ with $D_{e}=0$ may be traversed or not by the set of vehicles. The edge is called traversed edge if a vehicle traverses it without servicing.

The CARP meta-heuristic methodologies can be found in the excellent book by Dror [9]. In recent years, the most successful algorithms proposed in literature for CARP were based on different meta-heuristic models including, the tabu search algorithm of Hertz et al. [20], the variable neighborhood descent algorithm of Hertz and Mittaz [19], Polacek et al. [27], the guided local search heuristic of Beullens et al. [6], the memetic algorithm (MA) of Lacomme et al. [21], the evolution algorithm of Handa et al. [17, 18], the deterministic tabu search algorithm of Brandao and Eglese [7], the MA with extended neighborhood search of Tang et al. [30], and the ant colony optimization of Santos et al. [28, 29]. Recently, Bartolini et al. [2] proposed an improved lower bounds and exacts algorithm for CARP.

Comparing with the CARP, the split-delivery capacitated arc routing problem (SDCARP) can be treated as a relaxed constraint problem from the CARP [10, 11]. Given a fleet of vehicles capacity $W$, the object is to find a set of vehicle routes of minimum cost, such that each required edge is serviced by one or serval vehicles, each route starts and ends at a prespecified vertex $v_{0}$ (the depot) in $V$ and the total demand serviced by a route does not exceed the vehicle capacity $W$. The SDCARP was first introduced by Dror and Trudeau $[10,11]$ and the literature on SDCARP was much less than the CARP. Mullaseril et al. [26] proposed an adapted local search to solve the SDCARP, Guéguen [16] described a method of transforming the problem into a node-routing model. Labadi [23] introduced a memetic algorithm, and Belenguer et al. [5] proposed the lower bound and multi-start of iterated local search for SDCARP. The CARP and SDCARP were applied on postal deliveries, urban waste collection, and winter gritting.

As both problems are NP-hard, most of the methodologies applied to them are of a combinatoric nature, mainly using meta-heuristics. However, the CARP and SDCARP so far are solved as two distinct problems, and no attempt has been made to construct solutions from the relationship between them to our knowledge. The motivation of this paper is to contribute to the study of the SDCARP associated with the CARP. The paper is organized as follows. Section 2 presents the links between the two problems and introduces a intermediate model: UBO-CARP. The main components and the general structure of our algorithm, called crossover iterated local search, are described in section 3. Section 4 is devoted to computational experiments. The results obtained by the meta-heuristic are outperformed most of the existing best known solutions for the CARP on 63 instances of three sets. Some conclusions are given in section 5.

\section{A Construction Method from CARP to SDCARP Solution}

This section shows the connection between CARP and SDCARP. An intermediate model is used to transfer CARP to SDCARP and build new solutions. Compared 
with CARP, the split-delivery solution shows its advantage on both the total cost and the utilization rate of vehicles.

Figure 1 depicts a simple instance, where node 1 represents the depot, all three task-edges with cost 1 and $D(1,2)$ equals $2, D(2,3)$ and $D(2,4)$ equal 3 . The solution trips only consist of serviced edges. For the SDCARP, an additional list is used to represent the serviced demand. Given capacity 5 , the best cost of the problem is 8 with 2 routes: one vehicle servicing $(1,2)$ and a 3 demand edge, another servicing the last edge. When the capacity equals 4 , the cost is 10 with 3 vehicles: one of which services edge $(1,2)$ and the others service each of the remain edges. For the SDCARP, the cost is 8 with 2 vehicles, both service a unit demand of edge $(1,2)$ and one of the remain edges.

In the instance above, it can be seen that the cost of the best CARP solution is more than SDCARP when the capacity equals 4 . By relaxing the capacity constraint, we have tried to set up a new fleet with 2 vehicles in indirection: one vehicle services a 3 demand edge and the other vehicle services both $(1,2)$ and the last 3 demand. The latter vehicle services total 5 demand and exceeds its capacity. However, the cost is 8 , less than the best solution in CARP. In this paper, we refer to a solution as UBO-CARP solution if it violates the capacity constraint but provides an optimal cost.

Note that the UBO-CARP solution in this instance performs the same full paths of the SDCARP optimal solution, the second vehicle traverses edge $(1,2)$ and 1 capacity remains. As shown in Fig. 2, a unit of the demand in the first trip on edge $(1,2)$ can be distributed to this vehicle such that all trips can service 4 demand. Therefore, the solution could be deduced to an SDCARP solution of which the cost is 8 , less than the optimal solution of CARP.

Because the UBO-CARP performs a solution of lower cost than the CARP optimal solution, the cost would be reduced if it could be turned into an SDCARP. As each required edge in SDCARP can be serviced more than once, the demand of
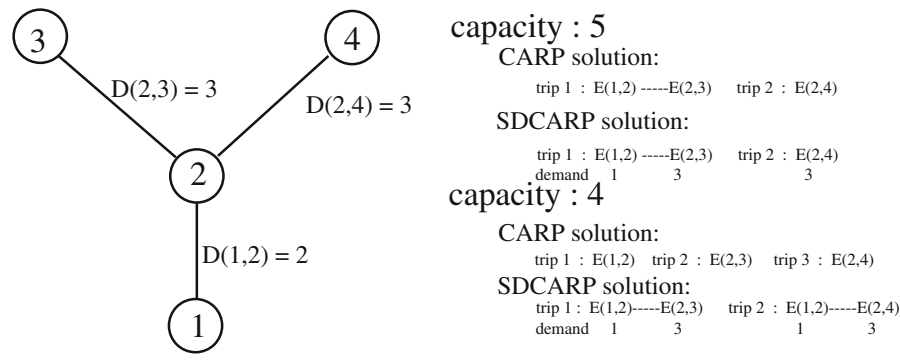

Fig. 1 CARP instance with 3 required edges
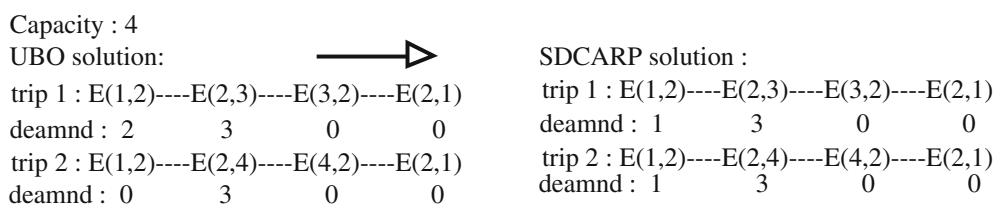

Fig. 2 Full path of UBO-CARP to SDCARP solution 
split-delivery edges can be aggregated and reassigned to the same trip. A simple proof shows that it obtains a CARP best solution or a UBO-CARP solution such that each best SDCARP solution can be linked with a CARP solution or an unsatisfied capacity constraint one with its cost less or equal than the best solution for CARP.

Proof Let $S$ represents the SDCARP solution making up with $N_{v}$ trips, where $N_{v}$ is the number of vehicles of capacity $W$. Let $N_{s}$ be the total amount of split-delivery edges. The SDCARP equals to a CARP solution when $N_{s}$ equals to 0 . If $N_{s} \geqslant 1$, we begin from the same split-delivery edges and then add and reallocate the total demand to one of them. Therefore, the service demand of other edges becomes 0 . As a result, we get a solution with $N_{s}-1$ split-delivery edges and so on. Finally, the result belongs to a solution of the CARP if it satisfies the capacity constraint, otherwise a UBO one.

Thanks to the property, finding the SDCARP solution can be treated as a two-step optimization problem. First, the UBO-CARP solution trips including serviced edges and traversed edges are computed by some heuristics. Then the split-delivery edges are constructed by reallocating the demand of required edges. An SDCARP solution is formed once the allocation policy satisfies the capacity constraint, of which the cost is less or equal than the best CARP solution. Note that a UBO-CARP solution is not always useful. Considering an optimal solution with one trip servicing all the edges in the instance, it can be easily checked that the solution satisfies all the relaxation constraints of UBO-CARP, but obviously it cannot be reconstructed. Thus, a parameter $F$ is used to limit the overload demand of each trip, the value of $F$ depends on the traversed times and the total demand of required edges in the trip. In the same instance, $F$ of each trip may be different. To simplify the calculation, we let $F$ be the maximum one in all trips, as a result, the capacity of vehicles are extended.

Let $T$ be the index set of all trips. For each trip $T_{i}$, let $X_{e}$ be the edge $e \in E_{R}$ serviced by trip $T_{i}$, and $Y_{e}$ be the edge $e \in E$ traversed by trip $T_{e}$. We denote by $S\left(R_{i}\right)$, or simply $S_{i}$, the set of required edges serviced by $T_{i}$. Let $C_{e}$ be the cost of $e$ in trip $T_{i}, X_{e}$ and $Y_{e}$ be two binary variables equal to 1 if and only if edge $e$ in trip $T_{i}$ is crossed in the solution. Note that the minimum number of vehicles needed to service the total demand is $K^{*}=\left[\Sigma_{e \in E_{R}} q_{e} / Q\right]$ [23]. The UBO-CARP can be formulated as the following LP model.

$$
\begin{gathered}
Z(S P)=\min \sum_{e \in T}\left(C_{e} \cdot X_{e}+C_{e} \cdot Y_{e}\right) \\
\text { s.t. } \quad \sum_{e \in T} D\left(X_{e}\right)=D_{e}, \forall e \in S_{i} \\
\sum_{e \in T} X_{e} \geqslant K^{*} \\
X_{e}, Y_{e} \in\{0,1\}, \forall e \in T
\end{gathered}
$$


The objective function (1) is the total cost traveled by the fleet of vehicles. Constraint (2) is a condition imposing that the total requirement of one edge is serviced by different vehicles in $E_{R}$. We assume that the amount of the service demand of a required edge is at least 1 . Constraint (3) imposes the lower bounds of vehicle numbers to service edge $e$.

\section{Crossover Iterated Local Search for the SDCARP}

\subsection{Related Meta-heuristics}

This section describes the feature of our algorithms, called crossover iterated local search (ILS). It is an algorithm based on ILS, but has a completely different search structure, as it requires an evolution method proposed in memetic algorithm (MA) to determine the beginning of each iteration. Therefore, every iteration starts from fully reproducible initial solutions. In this section, we first introduce the general structure of ILS and MA. Then we summarize the components of the two algorithms and show the combined structure of CO-ILS.

Iterated local search is a local search based meta-heuristic [24] and the search strategy is illustrated in Algorithm 1. ILS begins with an initial solution $S$ and uses a local search procedure to convert the solution into the local optimum $S^{*}$. Then, in each iteration, the current best solution is copied and moved to $S_{\text {new }}$ using a perturbation procedure and improved to $S_{\text {new }}^{*}$ by local search. Here, the best solution is replaced only in case of improvement. A survey of ILS can be found in [24].

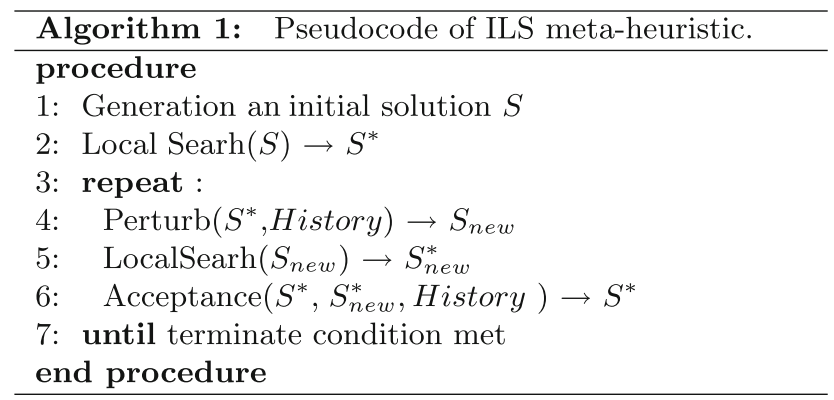

Memetic algorithm is an evolution heuristic introduced by Moscato [25], which can be viewed as a combination of population-based hybrid genetic algorithm (GA) and an individual learning procedure. As sketched in Algorithm 2, the procedure consists of chromosome evaluation, evolution operators and population management. First, an initial generation population is constructed by some heuristics and then comes the main evolution loop. In each generation, the population is evaluated and evolved using stochastic search and crossover procedure. Then a portion of the generation is randomly selected and improved using a search procedure. The loop is repeated until one terminate condition is met. The applications of MA are covered on a number of well-known 
combinatorial optimization problems, the typical use is proposed by Lacomme [21] in an arc routing problem. To search the solution space more efficiently, K. Tang et al. [30] introduced a memetic algorithm with extended neighborhood search (MAENS), of which a novel local search operator called merge-split (MS) is used.

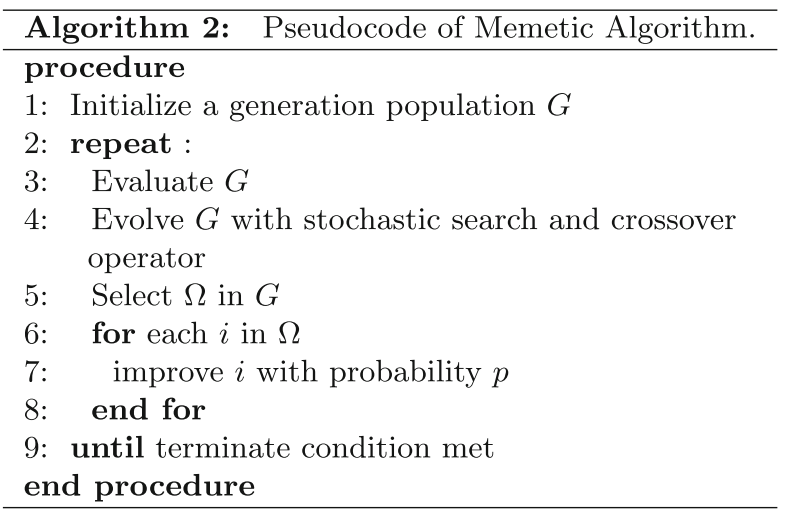

\subsection{General Structure of the Algorithm}

The CO-ILS algorithm is an ILS-based algorithm combined with MA. However, some components of other algorithms are also recycled. The CO-ILS reuses the extended path-scanning (EPS) heuristic [4, 28] and random arc selection (RS) heuristic [21] to compute the initial solutions. The evolution strategy in MA is reused to compute a new start of iteration. However, it differs from other local search based heuristic as it requires a crossover procedure instead of perturbation procedure in ILS, and it calls a specifical procedure to check solutions. Furthermore, a natural idea for the chromosomes in MA is to use giant tours, and separated by the partitioning procedure called trip delimiters. COILS mostly deals with the complete solutions. The solutions are encoded as giant tours only in crossovers. The CO-ILS consists of these four follow steps:

1. Generating initial solutions: Three methods are used to generate the initial solutions. Initial candidates are stored in set $U$ and randomly selected.

2. Local search strategy: The initial solution is converted into a local optimum by local search moves. Here, moves that violate the capacity constraint are allowed in a reasonable range. In general, the results of local search procedure may not feasible to CARP as they ignore the conditions. In fact, this step obtains a sequence of extended capacity CARP solutions (UBO-CARP).

3. Adjustment of the unsatisfied solutions: This step mainly deals with the UBO solutions. The demand of some edges is divided and redistributed to decrease the number of overload trips. The fleet routes that satisfy the capacity constraint drive to new SDCARP solutions.

4. Replacement and restart: A new start of local search iteration comes from crossing over a known solution $P_{1}$ with an initial one $P_{2}$ in set $U$. Then, step 2-4 are repeated until one of the termination conditions is satisfied. 


\subsection{Generating Initial Solutions}

The initial solution is created by three heuristic. Here, the total demand of one trip exceeding the capacity is allowed, but the demand should be less than $F$, where $F$ represents the upper bound of overload capacity. Moreover, a parameter $N_{l}$ is designed to limit the maximum amount of overload vehicles and split-delivery is not allowed in initialization.

\section{Random arc selection (RS) heuristic (Belenguer et al. [4], Santos et al. [29])}

The heuristic builds one trip at a time. In constructing each trip, the next arc to be added to the trip is randomly selected from the candidates who are not serviced at this point, only arcs with demand that will not exceed the remaining extended capacity are considered. A new route is started, if no such arc exists.

\section{Extended path-scanning (EPS) heuristic [21]}

The heuristic builds one trip at a time. Let $Q_{r}$ be the sorted queue of required edges which are not yet serviced. Each trip starts from the depot node and extends by adding arcs from $Q_{r}$ until it exceeds the expanded capacity. Five rules are used to sort the candidate arcs in EPS:

(i) to maximum the distance between current edge and the depot, (ii) to minimum the distance, (iii) to maximum the value of $D(e) / W(e)$, (iv) to minimum of the value, (v) if the capacity of vehicle is less than half-full then use rule i, else, use rule ii.

\section{Edge insertion (EI) heuristic}

The heuristic builds all the trips once a time. The EI begins with empty trips. In each iteration, a required edge which is not yet serviced is inserted into the best feasible position of all trips. However, the last insertion of one trip may exceed the capacity. It is allowed and the number of violating insertion should not above the limit $L$.

\subsection{Local Search Moves}

Five types of moves are used in our local search procedure and the details are shown below. In this local improvement procedure, moves are performed in a loop and each iteration is terminated with the first move that improves the current solution. To accelerate computation, a binary matrix $M_{k}(i, j)$ is created to record the trace of each iteration, where $M_{k}(i, j)$ represents the $K_{\text {th }}$ move of edge $(i, j), 0$ stands for the move has not done before, and 1 means the opposite. During the local search, $M_{k}(i, j)$ is marked with 1 if the $K_{\text {th }}$ move has done without improvement, otherwise, all positions in row $i, j$ and column $i, j$ of $M_{k}$ are marked with 0 . Moves are repeated until each element of $M_{k}$ equals 1 . Here, moves can violate the vehicle capacity and the maximum demand of overload moves is limited to $F$. 
Move 1: Single reverse, replace edge-task $e$ by its reversed edge rev $(e)$.

Move 2: Segment reverse, replace the segment between two position by the reversed segment.

Move 3: Swap, swap two edge-tasks $e_{1}$ and $e_{2}$, and the respective reversed edge-tasks. Move 4: Insertion, remove an edge-task from its current position and re-insert into another position of the solution.

Move 5: 2-opt move, swap two adjacent edges to another pair in a distinct trip, and the respective reversed edges.

\subsection{Adjust the Local Search Results}

In this section, we check every local search result as they may violate the capacity constraint. A procedure called adjustment is given to show how the UBO-CARP solutions can be transformed to SDCARP or CARP solutions and a dynamic model is used to reduce the amount of each overload trip. In all steps of the procedure, reversed edges are considered. The result obtains an SDCARP solution if it satisfies the constraints.

As shown in Algorithm 3 and 4, by adding all the traversed edges between each two adjacent serviced edges, the list $T$ of solution trips is extended. Let $Q$ be the new list presenting the serviced demand of each edge in $T$. Thus, each solution is coded as two corresponding lists $T$ and $Q$. Let $O$ be the set of the total trips which are not yet selected. The trips are ordered in $O$ with their demand. Let the largest one in $O$ be $t_{\text {obj }}$ which represents the current objective trip. Beginning from finding the same edge $e$ in $t_{\mathrm{obj}}$ and trip $t_{i}$ in $O \backslash t_{\mathrm{obj}}$, each iteration calculates the maximum split-delivery demand $D(e)$ of $e$ in $t_{\mathrm{obj}}$ that can be allocated to the other trip. A splitdelivery solution is found, if $D_{e}$ is larger than the overload demand. Otherwise, let the trip $t_{i}$ be the new object, $O:=O \backslash t_{i}$, this step is repeated until $O$ is empty.

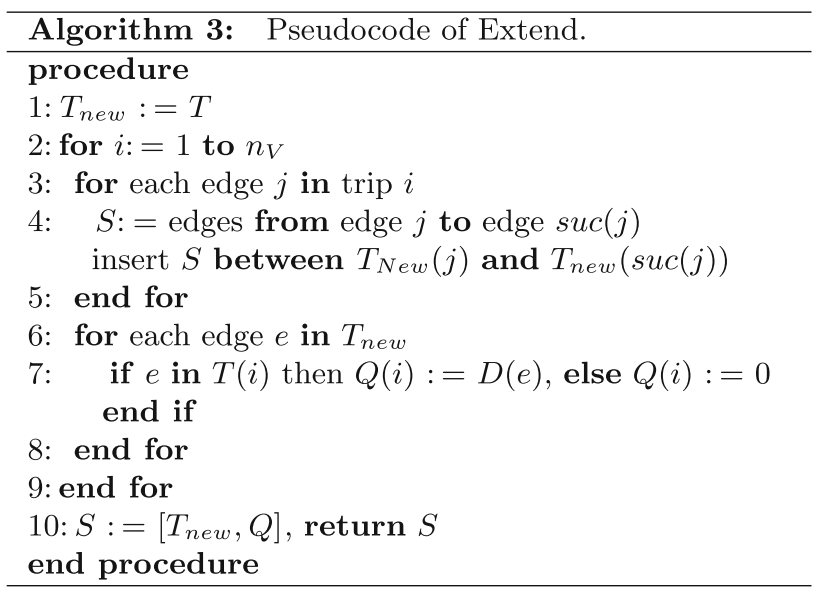




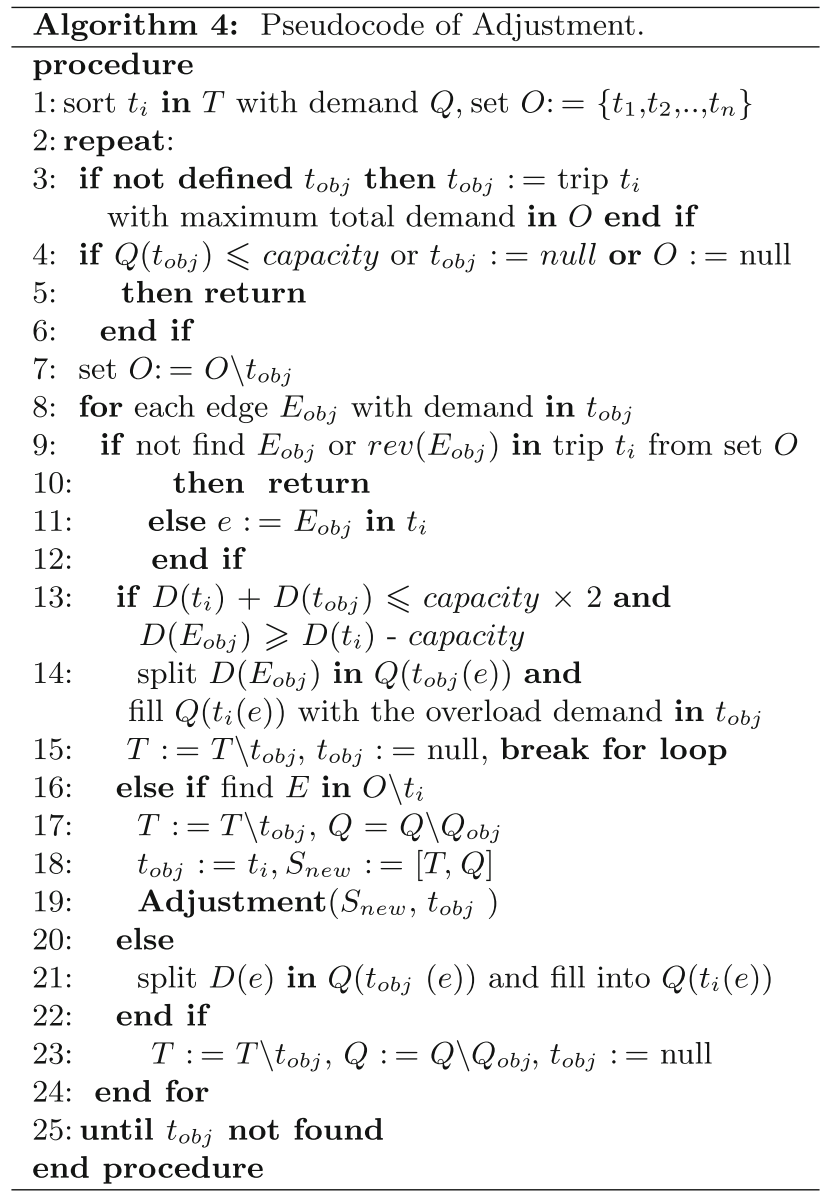

\subsection{Restart with Crossover}

Instead of the traditional way of perturbation procedure in iterated local search optimal method, we encode the solutions and crossover two solutions to perform a new start of each iteration. First, one local optimum is encoded as parent 1 . Then a better chromosome is selected by binary tournament method from set $U$ and encoded as parent 2 . We crossover them by LOX procedure [21] to generate a child chromosome and disaggregate the chromosome into serval feasible trips using a splitting procedure. At last, we update $U$.

A detailed introduction can be found in memetic algorithm of Lacomme. Let parent 1 and parent 2 be two parents chromosomes with total $N_{E r}$ require tasks. To get a child $C$, the parents are encoded as two lists and each procedure draws two random cutting sites $p$ and $q$ in the lists. First, LOX uses $P 1(p), \cdots, P 1(q)$ to fill $C(p), \cdots, C(q)$ and marks $\operatorname{missed}(p)$ to $\operatorname{missed}(q)$ with 1 in the edge tasks of $C$. Then $P 2$ is copied into $C(1), \cdots C(p-1)$ and $C(q+1), \cdots, C\left(N_{E r}\right)$ from 
left to right, and only edge task $e$ with $\operatorname{missed}(e):=0$ are in our consideration. In the steps, the missed $(e)$ equals 1 in $C$ if both $e$ and $\operatorname{rev}(e)$ are not yet in $C$. Algorithm 5 is the pseudocode of LOX, implemented as a function of two parents parent 1 , parent 2 that returns a child $C$. However, Algorithm 5 differs from that in Lacomme's as the result is partitioned by Ulusoy's splitting procedure (see in [31]).

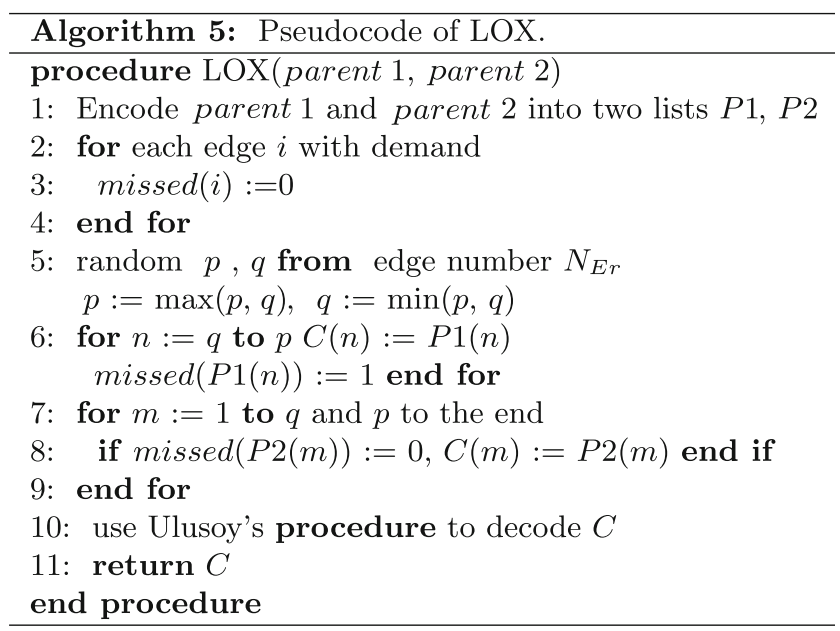

\subsection{General Structure of the CO-ILS}

The network of vehicles, arcs, and the depot is encoded using a reduced method. The depot node and the required edges and their reversed edges (if can be reversed) are stored in a matrix. We use Dijkstra's algorithm to compute a matrix $D_{i j}$ of shortest length between edge $i$ and $j$. By this trick, a trip can be encoded as a list of feasible required edges, with shortest paths between them. To accelerate computations, a single list is used in initial solution. However, in adjustment procedure, we extend the list with traversed edges and create a new list to record the split-delivery demand of each required edge. Thus, solutions can be encoded as two parallel lists: the first list $T$ represents the servicing trip and the second list $Q$ represents the delivery demand. The framework of crossover iterated local search for SDCARP is sketched below, including an initialization procedure. In initialization, we use different cost of the chromosomes to make the population diverse. 


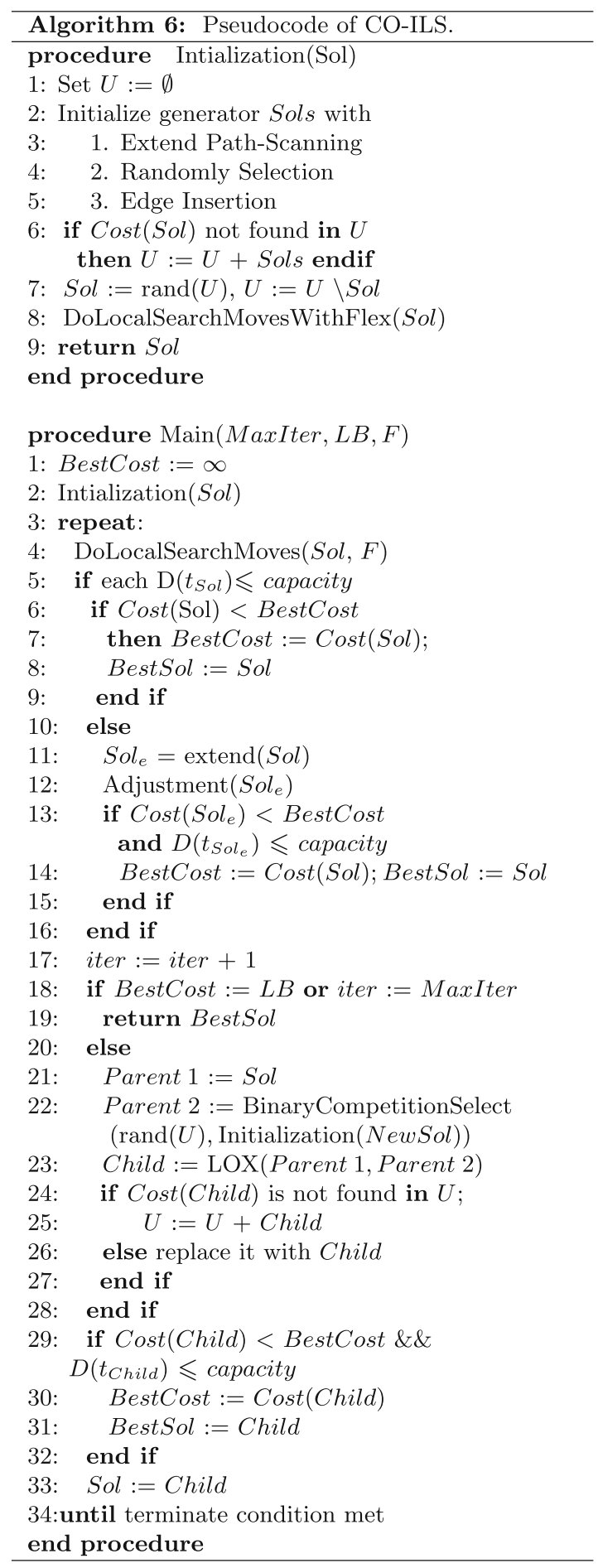




\section{Computational Result}

The CO-ILS runs on a 2.4-GHz PC with windows 7 Pro. The algorithm is tested on 63 instances of three sets in standard CARP instances. The data files can be downloaded at http://www.uv.es/ belengue/carp.html.

Two different approaches have been involved in our computational experiments, named SDCARP CO-ILS and CARP CO-ILS, representing respectively the two arc routing problems. The results are compared by total cost and running time. The CARP current best-known solutions are obtained in Ant-CARP meta-heuristics [27]. Each instance in table reports mean values for multiple runs of the heuristic. In the table below, we present the cost in bold, if we find a better cost of SDCARP.

Depending on whether the adjustment strategy is used and the value of $F$ we defined, the results are divided into two versions: the SDCARP and the traditional CARP solutions. In the first one, we allowed each local search iteration violating the vehicle capacity and run the adjustment procedure to rectify the solutions. We have tried to solve some instances with different $F$, where the value of $F$ was identified by hand. As each iteration may not find a feasible solution, we have run the algorithm several times until an available SDCARP solution was found. In the second one, all moves are satisfied the capacity constraint during our local search procedure, it can be viewed as an algorithm for CARP.

Considering the scale and average demands of the three sets, the following parameters were selected for the CO-ILS on the instances: Maxiter: $=50$ iterations, nRestart $:=20$ generations, and $F$ was initialized to 0 for the gdb and val files but 10 for the kshs files. However, the $F$ was added or reduced that depends on whether a valid solution has been found earlier. In computation, the first $75 \%$ restarted from a random initialization and the last part began with a local optimum solution obtained in the previous iterations. Note that the existing differences between the two arc routing problems may influence the results, the solutions are compared, respectively: one named CARP CO-ILS, the other SDCARP CO-ILS.

Table 1 gathers the results for gdb files. The table format is shared by all the results of data sets. First, the table shows the data file names, then the number of nodes $n$ and the number of edges $\tau$. The lower bounds $L B$ and the best-known solutions $B K S$ of the CARP benchmark are given in column 4 and 5. After that, we give the results of our algorithm in the next two columns: the CARP CO-ILS and the SDCARP CO-ILS. The average running time of the two versions is sketched in the last column. The deviation to the lower bound is compared in the bottom of the table. The cost is in bold, if it is better than the best known solution of CARP.

In these three sets of problems, all the edges of graphs are required edges. The first set is called gdb data files with small average demands and contains 23 instances generated by Armon and discussed by Golden et al. [14]. On the gdb instances, CO-ILS solves 21 of 23 instances to optimality and the SDCARP CO-ILS finds one better solution (gdb12) than the CARP solution. Compared with the CARP, the average deviation of the SDCARP to the lower bound reduced from 0.13 to $0.10 \%$. Due to the rather small demands, the CARP version is able to find optimal solutions without split deliveries. 
Table 1 Results for gdb data files

\begin{tabular}{|c|c|c|c|c|c|c|c|c|}
\hline \multirow[t]{3}{*}{ File } & \multirow[t]{3}{*}{$n$} & \multirow[t]{3}{*}{$\tau$} & \multirow[t]{3}{*}{ LB } & \multirow{3}{*}{$\begin{array}{l}\text { BKS } \\
\text { CARP }\end{array}$} & \multirow{3}{*}{$\begin{array}{l}\text { COILS } \\
\text { CARP }\end{array}$} & \multirow{2}{*}{\multicolumn{2}{|c|}{$\frac{\text { COILS }}{\text { SDCARP }}$}} & \multirow[t]{3}{*}{ Time } \\
\hline & & & & & & & & \\
\hline & & & & & & Average & Best & \\
\hline$g d b 1$ & 12 & 22 & 316 & 316 & 316 & 316.0 & 316 & 0.0 \\
\hline$g d b 2$ & 12 & 26 & 339 & 339 & 339 & 339.0 & 339 & 0.8 \\
\hline$g d b 3$ & 12 & 22 & 275 & 275 & 275 & 275.0 & 275 & 0.1 \\
\hline$g d b 4$ & 11 & 19 & 287 & 287 & 287 & 287.0 & 287 & 0.0 \\
\hline$g d b 5$ & 13 & 26 & 377 & 377 & 377 & 377.0 & 377 & 1.2 \\
\hline$g d b 6$ & 12 & 22 & 298 & 298 & 298 & 298.0 & 298 & 0.2 \\
\hline$g d b 7$ & 12 & 22 & 325 & 325 & 325 & 325.0 & 325 & 0.0 \\
\hline$g d b 8$ & 27 & 46 & 344 & 348 & 348 & 348.2 & 348 & 12.8 \\
\hline$g d b 9$ & 27 & 51 & 303 & 303 & 303 & 303.0 & 303 & 9.0 \\
\hline$g d b 10$ & 12 & 25 & 275 & 275 & 275 & 275.0 & 275 & 0.2 \\
\hline$g d b 11$ & 22 & 45 & 395 & 395 & 395 & 395.0 & 395 & 1.3 \\
\hline$g d b 12$ & 13 & 23 & 450 & 458 & 458 & 456.4 & 456 & 0.6 \\
\hline$g d b 13$ & 10 & 28 & 536 & 536 & 536 & 536.0 & 536 & 19.4 \\
\hline$g d b 14$ & 7 & 21 & 100 & 100 & 100 & 100.0 & 100 & 0.1 \\
\hline$g d b 15$ & 7 & 21 & 58 & 58 & 58 & 58.0 & 58 & 0.3 \\
\hline$g d b 16$ & 8 & 28 & 127 & 127 & 127 & 127.0 & 127 & 1.5 \\
\hline$g d b 17$ & 8 & 28 & 91 & 91 & 91 & 91.0 & 91 & 0.1 \\
\hline$g d b 18$ & 9 & 36 & 164 & 164 & 164 & 164.0 & 164 & 0.4 \\
\hline$g d b 19$ & 8 & 11 & 55 & 55 & 55 & 55.0 & 55 & 0.3 \\
\hline$g d b 20$ & 11 & 12 & 121 & 121 & 121 & 121.0 & 121 & 0.5 \\
\hline$g d b 21$ & 11 & 33 & 156 & 156 & 156 & 156.0 & 156 & 0.0 \\
\hline$g d b 22$ & 11 & 44 & 200 & 200 & 200 & 200.0 & 200 & 6.7 \\
\hline$g d b 23$ & 11 & 55 & 233 & 233 & 233 & 233.0 & 233 & 68.6 \\
\hline Average $\%$ & & & & 0.13 & 0.13 & 0.11 & 0.10 & \\
\hline Worst \% & & & & 1.78 & 1.78 & 1.42 & 1.33 & \\
\hline Optimal & & & & 21 & 21 & 21 & 21 & \\
\hline Best & & & & 22 & 22 & 22 & 23 & \\
\hline
\end{tabular}

The second test set named kshs with six instances, where the different instances have been created by changing the set of required edges and the numbers of the vehicles. The results of kshs instances are shown in Table 2 which reuses the table format of Table 1. On the kshs instances, CARP CO-ILS outperforms all bestknown solutions while SDCARP CO-ILS solves all of six instances to optimality and reaches $3.60 \%$ improvement on kshs4. Compared with CARP, the average deviation of the SDCARP to the lower bound reduced from 0.6 to $0.0 \%$, this can be explained by the increased average demands in kshs instances and the results of the split-delivery.

Table 3 shows the results on the val data files with 34 problems generated by Benavent et al. (1992). On these instances, CO-ILS solves 23 problems to their 
Table 2 Results for kshs data files

\begin{tabular}{|c|c|c|c|c|c|c|c|c|}
\hline \multirow[t]{3}{*}{ File } & \multirow[t]{3}{*}{$n$} & \multirow[t]{3}{*}{$\tau$} & \multirow[t]{3}{*}{ LB } & \multirow{3}{*}{$\begin{array}{l}\text { BKS } \\
\text { CARP }\end{array}$} & \multirow{3}{*}{$\begin{array}{l}\text { COILS } \\
\text { CARP }\end{array}$} & \multirow{2}{*}{\multicolumn{2}{|c|}{$\frac{\text { COILS }}{\text { SDCARP }}$}} & \multirow[t]{3}{*}{ Time } \\
\hline & & & & & & & & \\
\hline & & & & & & Average & Best & \\
\hline$k s h s 1$ & 8 & 15 & 14661 & 14661 & 14661 & 14661 & 14661 & 0.1 \\
\hline$k s h s 2$ & 10 & 15 & 9863 & 9863 & 9863 & 9863 & 9863 & 0.1 \\
\hline kshs3 & 6 & 15 & 9320 & 9320 & 9320 & 9320 & 9320 & 0.0 \\
\hline$k s h s 4$ & 8 & 15 & 11098 & 11498 & 11498 & 11138 & 11098 & 0.2 \\
\hline kshs5 & 8 & 15 & 10957 & 10957 & 10957 & 10957 & 10957 & 0.1 \\
\hline$k s h s 6$ & 9 & 15 & 10197 & 10197 & 10197 & 10197 & 10197 & 0.1 \\
\hline Average $\%$ & & & & 0.60 & 0.60 & 0.06 & 0.00 & \\
\hline Worst \% & & & & 3.60 & 3.60 & 0.36 & 0.00 & \\
\hline Optimal & & & & 5 & 5 & 5 & 6 & \\
\hline Best & & & & 5 & 5 & 5 & 6 & \\
\hline
\end{tabular}

optimality for CARP. For SDCARP, it finds 25 optimum and one better solution to its lower bound on vallc. Unfortunately, the running time on val2b, val9a and val10b is quite longer. We impose this shortcoming is the negative result of creating large scale of trip lists in our algorithm. In each local search iteration, the moves search the total trip lists which may increase the computing time. However, the algorithm performs well on other instances. The average deviation of the SDCARP to the lower bound is $0.30 \%$, and the worst reduced from 4.26 to $1.74 \%$.

\section{Conclusions}

To summarize, the best CO-ILS solutions for the CARP presented in the tables outperform most of the best-known solutions by other heuristics on three sets of available benchmarks in public. The CO-ILS with split-delivery finds three optimal solutions than traditional CARP solutions that are bold in the tables. SDCARP COILS reaches small gaps to lower bounds on all data sets. The performance results from a combination of iterated local search and memetic algorithm. To begin with, a few good initial solutions are obtained by three heuristics, each iterator is strongly improved by local search. Compared with memetic algorithm, our approach costs more time in one generation but provides higher quality child. The using of extended capacity search iterators makes it possible to find new solutions by traversing the infeasible zone; as a result, it searches more deeply and frequently. After local search, we use the adjustment strategy to ensure the feasible solutions. The large demand edges with frequently traveled by, is more likely to split. That is the reason why the solutions on relatively large demand problems, such as kshs4, is well-performing. During the crossover between distinct solutions, the superior chromosomes can be kept and the solution is converted into another solution space 
Table 3 Results for val data files

\begin{tabular}{|c|c|c|c|c|c|c|c|c|}
\hline \multirow[t]{3}{*}{ File } & \multirow[t]{3}{*}{$n$} & \multirow[t]{3}{*}{$\tau$} & \multirow[t]{3}{*}{ LB } & \multirow{3}{*}{$\begin{array}{l}\text { BKS } \\
\text { CARP }\end{array}$} & \multirow{3}{*}{$\begin{array}{l}\text { COILS } \\
\text { CARP }\end{array}$} & \multirow{2}{*}{\multicolumn{2}{|c|}{$\frac{\text { COILS }}{\text { SDCARP }}$}} & \multirow[t]{3}{*}{ Time } \\
\hline & & & & & & & & \\
\hline & & & & & & Average & Best & \\
\hline val1a & 24 & 39 & 173 & 173 & 173 & 173.0 & 173 & 0.2 \\
\hline $\operatorname{val} 1 b$ & 24 & 39 & 173 & 173 & 173 & 173.0 & 173 & 17.5 \\
\hline val $1 c$ & 24 & 39 & 235 & 245 & 245 & 236.0 & 235 & 5.8 \\
\hline val $2 a$ & 24 & 34 & 227 & 227 & 227 & 227.0 & 227 & 3.7 \\
\hline val $2 b$ & 24 & 34 & 259 & 259 & 259 & 259.0 & 259 & 4.4 \\
\hline $\operatorname{val} 2 \mathrm{c}$ & 24 & 34 & 457 & 457 & 457 & 457.0 & 457 & 60.3 \\
\hline$v a l 3 a$ & 24 & 35 & 81 & 81 & 81 & 81.0 & 81 & 0.6 \\
\hline val3b & 24 & 35 & 87 & 87 & 87 & 87.0 & 87 & 17.6 \\
\hline $\operatorname{val} 3 c$ & 24 & 35 & 138 & 138 & 138 & 138.0 & 138 & 16.8 \\
\hline val $4 a$ & 41 & 69 & 400 & 400 & 400 & 400.0 & 400 & 149.1 \\
\hline val $4 b$ & 41 & 69 & 412 & 412 & 412 & 412.0 & 412 & 35.9 \\
\hline val $4 c$ & 41 & 69 & 428 & 428 & 428 & 430.0 & 428 & 53.8 \\
\hline val4d & 41 & 69 & 526 & 530 & 531 & 531.0 & 531 & 0.6 \\
\hline val5a & 34 & 65 & 423 & 423 & 423 & 423.0 & 423 & 105.6 \\
\hline val $5 b$ & 34 & 65 & 446 & 446 & 446 & 446.0 & 446 & 63.9 \\
\hline val $5 \mathrm{c}$ & 34 & 65 & 470 & 474 & 477 & 475.6 & 474 & 72.5 \\
\hline val5d & 34 & 65 & 573 & 575 & 581 & 581.0 & 581 & 135.6 \\
\hline val $6 a$ & 31 & 50 & 223 & 223 & 223 & 223.0 & 223 & 48.0 \\
\hline val $6 b$ & 31 & 50 & 231 & 233 & 233 & 233.0 & 233 & 91.5 \\
\hline val $6 c$ & 31 & 50 & 313 & 317 & 317 & 317.0 & 317 & 34.9 \\
\hline val7a & 40 & 66 & 279 & 279 & 279 & 279.0 & 279 & 65.4 \\
\hline $\operatorname{val7b}$ & 40 & 66 & 283 & 283 & 283 & 283.0 & 283 & 26.0 \\
\hline val7c & 40 & 66 & 334 & 334 & 334 & 334.0 & 334 & 121.3 \\
\hline val8a & 30 & 63 & 386 & 386 & 386 & 386.0 & 386 & 96.4 \\
\hline val $8 b$ & 30 & 63 & 395 & 395 & 395 & 395.0 & 395 & 90.6 \\
\hline val $8 \mathrm{c}$ & 30 & 63 & 518 & 521 & 533 & 533.0 & 527 & 130.7 \\
\hline val9a & 50 & 92 & 323 & 323 & 323 & 323.0 & 323 & 767.2 \\
\hline val $9 b$ & 50 & 92 & 326 & 326 & 326 & 326.0 & 326 & 276.9 \\
\hline $\operatorname{val} 9 c$ & 50 & 92 & 332 & 332 & 332 & 332.0 & 332 & 155.6 \\
\hline val9d & 50 & 92 & 385 & 389 & 391 & 391.0 & 391 & 80.6 \\
\hline val10a & 50 & 33 & 428 & 428 & 430 & 430.0 & 428 & 180.8 \\
\hline val10b & 50 & 97 & 436 & 436 & 436 & 436.0 & 436 & 113.9 \\
\hline val $10 c$ & 50 & 97 & 446 & 446 & 448 & 448.0 & 448 & 50.3 \\
\hline val10d & 50 & 97 & 525 & 525 & 529 & 529.0 & 529 & 286.6 \\
\hline Average $\%$ & & & & 0.17 & 0.48 & 0.34 & 0.30 & \\
\hline Worst \% & & & & 4.26 & 4.26 & 2.90 & 1.74 & \\
\hline Optimal & & & & 26 & 23 & 22 & 25 & \\
\hline Best & & & & 33 & 26 & 26 & 29 & \\
\hline
\end{tabular}


to avoid converging prematurely. The superior parent chromosomes also can accelerate computation in the next local search procedure so that the total number of crossovers is decreased. Moreover, the SDCARP solutions perform higher utilization rate of vehicles and also this paper reflects the increasing research activity on split-delivery capacity arc routing problems [1, 3, 8, 12, 15, 22].

\section{References}

[1] Amberg, A., Domschke, W.: Multiple center capacitated arc routing problems: A tabu search algorithm using capacitated trees. Eur. J. Oper. Res. 124(2), 360-376 (2000)

[2] Bartolini, E., Cordeau, J.F., Laporte, G.: Improved lower bounds and exact algorithm for the capacitated arc routing problem. Math. Progr. 137(1), 409-452 (2013)

[3] Belenguer, M., Benavent, E.: A cutting plane algorithm for the capacitated arc routing problem. Comput. Oper. Res. 30(5), 705-728 (2003)

[4] Belenguer, M., Benavent, E., Lacomme, P., et al.: Lower and upper bounds for the mixed capacitated arc routing problem. Comput. Oper. Res. 33(12), 3363-3383 (2006)

[5] Belenguer, M., Benavent, E., Labadi, N., et al.: Split-delivery capacitated arc-routing problem: lower bound and metaheuristic. Trans. Sci. 44(2), 206-220 (2010)

[6] Beullens, P., Muyldermans, L., Cattrysse, D., et al.: A guided local search heuristic for the capacitated arc routing problem. Eur. J. Oper. Res. 147(3), 629-643 (2003)

[7] Brandao, J., Eglese, R.: A deterministic tabu search algorithm for the capacitated arc routing problem. Comput. Oper. Res. 5(4), 1112-1126 (2008)

[8] Chu, F., Labadi, N.: A scatter search for the periodic capacitated arc routing problem. Eur. J. Oper. Res. 169(2), 586-605 (2006)

[9] Dror, M.: Arc Routing, Theory, Solutions and Applications. Springer, New York (2000)

[10]Dror, M., Trudeau, P.: Savings by split delivery routing. Trans. Sci. 23(2), 141-145 (1989)

[11]Dror, M., Trudeau, P.: Split delivery routing. Naval Res. Log. 37(3), 383-402 (1990)

[12]Filippi, C., Alberto, D.P.: A variable neighborhood descent algorithm for a real waste collection problem with mobile depots. Int. Trans. Oper. Res. 13(2), 125-141 (2006)

[13]Golden, B.L., Wong, R.T.: Capacitated Arc Routing Problems. Networks 11(3), 305-315 (1981)

[14]Golden, B.L., DeArmon, J., Baker, E.K.: Computational experiments with algorithms for a class of routing problems. Comput. Oper. Res. 10(1), 47-59 (1983)

[15]Grard, F., Lacomme, P., Prins, C.: Evolutionary algorithm for stochastic arc routing problems. Appl. Evol. Comput. 3005, 501-512 (2004)

[16]Guguen, J., Wagner, J.R.: Surface functional properties of native, acid-treated, and reduced soy glycinin 2 emulsifying properties. J. Agric. Food Chem. 47(6), 2181-2187 (1999)

[17]Handa, H., Chapman, L., Yao, X.: Robust route optimization for gritting/salting trucks: A CERCIA experience. Comput. Intell. Mag. IEEE 1(1), 6-9 (2006)

[18]Handa, H., Lin, D., Chapman, L., Yao, X.: Robust solution of salting route optimisation using evolutionary algorithms, Evolutionary Computation, 2006. CEC 2006, IEEE Congress, Vancouver, Canada, pp. 3098-3105.

[19]Hertz, A., Mittaz, M.: A variable neighborhood descent algorithm for the undirected capacitated arc routing problem. Transp. Sci. 35(4), 425-434 (2001) 
[20]Hertz, A., Laporte, G., Mittaz, M.: A tabu search heuristic for the capacitated arc routing problem. Oper. Res. 48(1), 129-135 (2000)

[21]Lacomme, P., Prins, C., Ramdane-Cherif, W.: Competitive memetic algorithms for arc routing problems. Ann. Oper. Res. 131(1), 159-185 (2004)

[22]Lacomme, P., Prins, C., Senaux, M.: A genetic algorithm for a bi-objective capacitated arc routing problem. Comput. Oper. Res. 33(12), 3473-3493 (2006)

[23]Labadi, N., Prins, C., Reghioui, M.: An evolutionary algorithm with distance measure for the split delivery capacitated arc routing problem. Recent advances in evolutionary computation for combinatorial optimization, pp. 275-294. Springer, New York (2008)

[24]Lourenco, H.R., Martin, O.C., Stutzle, T.: Iterated local search. In: Glover, F., Kochenberger, G. (eds.) Handbook of Metaheuristics, pp. 321-353. Springer, New York (2002)

[25] Moscato P.: On evolution, search, optimization, genetic algorithms and martial arts: towards memetic algorithms[R]. Technical Report C3P 826, Caltech Concurrent Computation Program, California Institute of Technology, Pasadena, USA, (1989).

[26]Mullaseril, P.A., Dror, M., Leung, J.: Split-delivery routing heuristics in livestock feed distribution. J. Oper. Res. Soc. 48(2), 107-116 (1997)

[27]Polacek, M., Doerner, K.F., Hartl, R.F., Maniezzo, V.: A variable neighborhood search for the capacitated arc routing problem with intermediate facilities. J. Heuristics. 14(5):405-423 (2008)

[28]Santos, L., Coutinho-Rodrigues, J.: An improved heuristic for the capacitated arc routing problem. Comput. Oper. Res. 36(9), 2632-2637 (2009)

[29]Santos, L., Coutinho-Rodrigues, J., Current, J.R.: An improved ant colony optimization based algorithm for the capacitated arc routing problem. Transp. Res. Part B: Methodol. 44(2):246-266 (2010)

[30]Tang, K., Mei, Y., Yao, X.: Memetic algorithm with extended neighborhood search for capacitated arc routing problems. IEEE Trans. Evol. Comput. 13(5), 1151-1166 (2009)

[31]Ulusoy, G.: The fleet size and mix problem for capacitated arc routing. Eur. J. Oper. Res. 22(3), 329-337 (1985) 